\title{
Elastic continuum theories of lattice defects: a review
}

\author{
DEBENDRANATH SAHOO \\ Materials Science Laboratory, Reactor Research Centre, Kalpakkam 603 102, India
}

\begin{abstract}
The presently available elastic continuum theories of lattice defects are reviewed. After introducing a few elementary concepts and the basic equations of elasticity the Eshelby's theory of misfitting inclusions and inhomogeneities is outlined. Kovács' result that any lattice defect can be described by a surface distribution of elastic dipoles is described. The generalization of the isotropic continuum approach to anisotropic models and to Eringen's isotropic but non-local model is discussed. Kröner's theroy (where a defect is viewed as a lack of strain compatibility in the medium) and the elastic field equations (formulated in a way analogous to Maxwell's field equations of magnetostatics) are described. The concept of the dislocation density tensor is introduced and the utility of higher-order dislocation density correlation tensors is discussed. The beautiful theory of the affine differential geometry of stationary lattice defects developed by Kondo and Kröner is outlined. Kosevich's attempt to include dynamics in the elastic field equations is described. Wadati's quantum field theory of extended objects is mentioned qualitatively. Some potential areas of research are identified.
\end{abstract}

Keywords. Elastic continuum theory; lattice defects; inhomogeneities; dislocations; disclinations; dislocation density tensor; torsion tensor; curvature tensor; dislocation density correlations; elastic field equations; nonlocal elasticity.

\section{Introduction}

This paper is intended to survey the elastic continuum theories of lattice defects. The understanding of the behaviour of crystal defects is essential for studying such processes as anelasticity, plastic flow, rupture, fatigue and radiation damage, which play a crucial role in materials science. The lattice distortion produced by a defect can be calculated by using elastic models at sufficiently large distances from the defect. In many applications, a continuum theory offers precise analytic expressions for the stress fields of defects, their interaction energies, etc., and hence is of immense practical value. In contrast to this, a discrete atomistic approach (which is unavoidable at short distances from the defects) does not offer analytically convenient working relations, although it yields reliable numerical data.

At present there is no unified theory of defects in continuous media. Most theories are developed with a particular type of defect in view. Thus the methods applicable to point defects are different from those applicable to line defects-for dislocations, see e.g., de Wit (1960), Nabarro (1967), Mura (1968); for volume defects (inclusions), see Eshelby (1957). Moreover, almost all theories are restricted to static defect problems. The dynamical theory of defects in continua is still in its infancy. This review presents a bird's eyeview of the various approaches to defects in a continuous medium. 


\section{Continuum modelling of defects}

In the elastic continuum theory, defects are modelled following the procedure given by (See e.g. Eshelby 1956). In order to construct a defect, one marks out in the medium an appropriate surface $S_{C}$ bounded by a curve $C$ and makes a cut coinciding with $C$. Then a relative displacement $\delta \mathbf{u}$ is given to each pair of points on either side of $S_{C}$, scraping away material where there would be interpenetration and filling in the remaining gaps with additional material. In general, $\delta u$ can be written as

$$
\delta u_{i}=b_{i}+\Omega_{i j} x_{j} \quad(i, j=1,2,3),
$$

where $x_{j}$ denotes the coordinates of the point, $b_{i}$ the relative translation of this point, and $\Omega_{i j}\left(=-\Omega_{j i}\right)$ describes the relative rotation of $S_{c}$. A general dislocation is thus described by six constants - in other words, it consists of six elementary (Volterra) dislocations. Each of the latter can be characterized by a single constant, the other five being set equal to zero. A rotational dislocation with $b_{i}=0$ is actually a disclination. When $\Omega_{i j}=0$, one has the usual (translational) dislocation with a Burgers vector $\mathbf{b}$. Some examples of defects created in this manner are shown in figure 1. Figure 1(a) shows an edge dislocation produced by cutting a ring-shaped cylinder across the surface $S_{C}$, choosing the axis of the cylinder as the curve $C$, and displacing the upper side of the surface $S_{C}$ over the lower by an amount b perpendicular to the axis. In figure 1(b), a sector of the cylinder is cut defining the surface $S_{C}$ and the axis as $C$, and both sides of the surface are pulled apart widening the angle of the sector so as to accommodate a larger sized sector. This produces a wedge disclination, $\boldsymbol{\Omega}$ being a rotation around the axis. Figure 1(c) shows the Somigliana prescription for creating a substitutional or an interstitial atom. A sphere is cut out of the matrix, its radius altered by adding or removing material, and the sphere is then reinserted in the matrix. This is the familiar misfitting sphere model. Note that there is no boundary curve $C$ in this case. It can be shown that a knowledge of $\delta \mathbf{u}$ as a function of position $x_{i}$ on the surface $S_{C}$, together with the boundary conditions at the surface of the body, completely determines the resulting state of internal stress.

\section{Basic equations of elasticity theory}

In the linear theory of elasticity, the condition of equilibrium is given by

$$
\sigma_{i h, l}+f_{i}=0 \text {, }
$$

where $\sigma_{i l}$ is the stress tensor, $f_{i}$ is the body force and the usual comma notation for derivatives and the convention of summation over repeated indices are used. $\sigma_{i j}$ is related to the strain tensor $e_{i j}$ by Hooke's law:

$$
\sigma_{i l}=C_{i l m n} e_{m n},
$$

where

$$
e_{m n}=\frac{1}{2}\left(u_{m, n}+u_{n, m}\right)
$$

is the symmetric part (sym) of the gradient (grad) of the displacement field $\mathbf{u}$ : thus $\boldsymbol{e}=\operatorname{sym} \operatorname{grad} \mathbf{u} \equiv \operatorname{def} \mathbf{u}$. For an isotropic homogeneous medium

$$
C_{i l m n}=\lambda \delta_{i l} \delta_{m n}+\mu\left(\delta_{i m} \delta_{l n}+\delta_{i n} \delta_{l m}\right),
$$



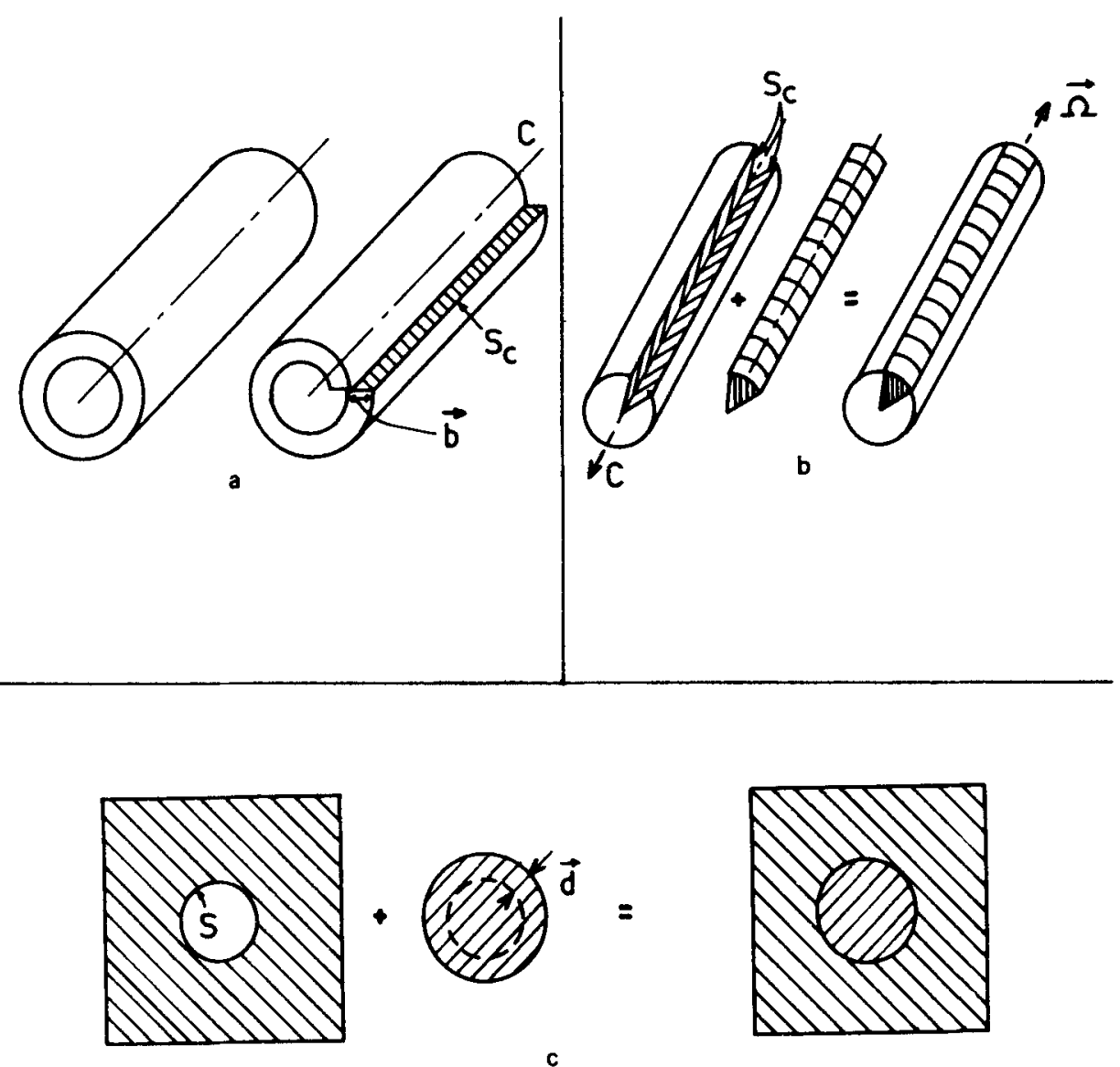

Figure 1. (a) Continuum model of an edge dislocation. (b) Continuum model of a wedge disclination. (c) Misfitting sphere model for a substitutional atom.

where $\lambda$ is Lame's constant and $\mu$ is the shear modulus. Equations (1)-(3) imply the differential equations

$$
C_{i l m n} u_{m, n l}+f_{i}=0
$$

for $\mathbf{u}$. Let $G_{l i}$ be the Green function tensor defined by the equation

$$
C_{i j k l} G_{k m, i j}(|\mathbf{r}|)+\delta_{i m} \delta(|\mathbf{r}|)=0
$$

and the boundary condition $G_{k m}(r \rightarrow \infty)=0$. Then

$$
G_{k m}(r)=\frac{1}{8 \pi \mu}\left[\delta_{k m} r_{, p p}-\frac{\lambda+\mu}{\lambda+2 \mu} r_{, k m}\right] \text {. }
$$

The elastic field due to the body force $f$ is given by

$$
u_{l}(\mathbf{r})=\int f_{i}\left(\mathbf{r}^{\prime}\right) G_{l i}\left(\left|\mathbf{r}-\mathbf{r}^{\prime}\right|\right) \mathrm{d}^{3} r^{\prime}
$$

The existence of a continuous single-valued displacement field $u_{i}$ requires the 
incompatibility (inc) of the strain tensor to vanish, i.e.,

$$
\text { inc } e=0 \text {, }
$$

where, in component form,

$$
(\text { inc } e)_{i j}=-\varepsilon_{i k l} \varepsilon_{j m n} e_{l n, k m}=[\operatorname{curl}(\operatorname{curl} e)]_{i j},
$$

$\varepsilon_{i k l}$ denoting the completely antisymmetric Levi-Civita tensor or the alternator.

There are two broad ways of introducing defects into this picture. One is to start with (1) and assign an effective $f_{i}$, as is usually done in a point defect (Eshelby 1957). In line defects, one puts $f_{i}=0$, but generalises $(8)$ to include the contribution from the cut surface $S_{C}$ (Mura 1968). In the second approach, one must recognise that when defects are present in the medium, the displacement field $\mathbf{u}$ is no longer a continuous singlevalued function on $S_{C}$, and therefore the compatibility condition ought to be modified. This is achieved by generalizing (9) to include a source term $\eta$ characterising the incompatibility distribution of the defect, i.e., (9) is replaced by

$$
\text { inc } e=\eta \text {. }
$$

In the next section we describe briefly the first approach which is due largely to Eshelby (1957).

\section{The Eshelby approach}

Volume defects such as precipitates, solute atoms, self-interstitials, etc contribute to the change in the elastic state of the medium. A self-interstitial is modelled as a misfitting homogeneous inclusion (MHI). It corresponds to matter of volume $V+\Delta V$ being forced into a cavity of volume $V$ in the medium (see figure $2 a$ ). Both the inclusion $(I)$ and the matrix $(M)$ have the same elastic moduli. An MHI is created in a body because of internal stress, i.e., some region $I$ of volume $V$ develops internal stress and is transformed to a different shape and size with a volume $V+\Delta V^{c}$ while remaining inside the matrix $M$ (see figure $2 \mathrm{~b}$ ). Thus a constrained stress $\left(\sigma_{i j}^{C}\right)$ or a constrained strain $\left(e_{i j}^{C}\right)$ is developed both in $I$ and $M$.

Now suppose $M$ was absent and $I$ alone were to undergo a transformation (see figure 2c). It would acquire a different shape and size, with volume $V+\Delta V^{T}$. Let $\sigma_{i j}^{T}$ and $e_{i j}^{T}$ be the stress and strain associated with the transformation. These need not be of elastic origin (they may arise from metallurgical transformations, for example). Thus, symbolically, $I \stackrel{T}{\rightarrow} I^{T}$, and $M \stackrel{T}{\rightarrow} M$. Eshelby has posed and answered the following questions:

(i) How can one relate $\sigma_{i j}^{C}$ and $e_{i j}^{C}$ to $\sigma_{i j}^{T}$ and $e_{i j}^{T}$ ? (ii) What can one deduce about the behaviour of $\mathbf{u}^{C}, \sigma^{C}$ and $e^{C}$, the dilation and the self-energy of an MHI of arbitrary shape? (iii) What is the interaction energy of an $\mathrm{MHI}$ with an applied stress field $\sigma_{i j}^{A}$ ?

After answering these questions Eshelby asks the same questions for an "inhomogeneity" (see figure 2d). The inhomogeneity is an inclusion having elastic constants $\left(\lambda^{*}, \mu^{*}\right)$ different from those of the matrix $(\lambda, \mu)$. Note that a perfectly-fitting inhomogeneity (PFI) does not perturb the stress field in the matrix. However, a misfitting inhomogeneity (MFI) would alter the stress field in $M$.

An MFI is an appropriate model for a foreign interstitial in a crystal. Eshelby shows 
how to reduce the problem of an MFI to an equivalent MHI problem. The problem of an MFI in the presence of an external stress $\sigma_{i j}^{A}$ is of interest in many practical situations such as SIPA creep (Heald and Speight 1974), voids in strained metals, etc. In the presence of $\sigma_{i j}^{A}$, a PFI can undergo an induced transformation strain $e_{i j}^{T}$ 'proportional' to the applied strain $e_{i j}^{A}$. To illustrate the Eshelby approach, consider an MHI for which the displacement field $u_{i}^{C}$ is given by

$$
u_{i}^{C}(r)=\int \mathrm{d} S_{l}^{\prime} C_{j l m n} e_{m n}^{T}\left(\mathbf{r}^{\prime}\right) G_{i j}\left(\left|\mathbf{r}-\mathbf{r}^{\prime}\right|\right) .
$$

Eshelby considers a spherical and an ellipsoidal MHI for which $\boldsymbol{e}_{m n}^{T}$ is constant, and, using (7) for the Green function, arrives at the familiar relations (for the spherical MHI):

$$
\begin{aligned}
e_{i j}^{C} & ={ }^{\prime} e_{i j}^{C}+\frac{1}{3} e^{C} \delta_{i j} \\
& =\beta^{\prime} e_{i j}^{T}+\frac{1}{3} \alpha e^{T} \delta_{i j}, \\
e^{C} & \equiv e_{i i}^{C}, e_{i j}^{C} \equiv e_{i j}^{C}-\frac{1}{3} e^{C} \delta_{i j}, \\
\alpha & =\frac{1}{3}\left(\frac{1+v}{1-v}\right), \beta=\frac{2}{15}\left(\frac{4-5 v}{1-v}\right) .
\end{aligned}
$$

Here ' $e_{i j}$ is the deviatioric part of $e_{i j}$ and $e$ is the scalar part. The general results for a PFI and an MFI under an applied load are given in Eshelby (1957).

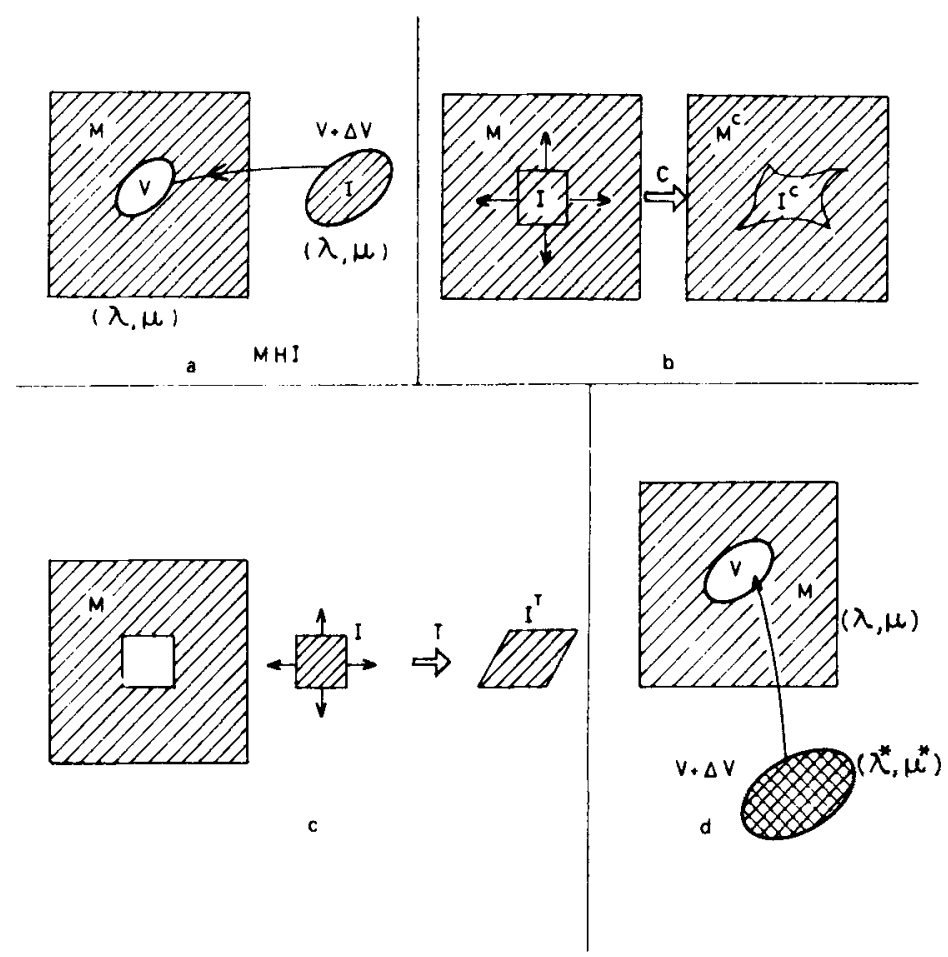

Figure 2. (a) A misfitting homogeneous inclusion (MHI). (b) The rectangular region I undergoing a constrained transformation in the matrix resulting in a curved shape. (c) The region $I$, detached from the matrix, undergoing a $T$ transformation. (d) A misfitting inhomogeneity (MFI). 
A significant feature of Eshelby's theory is the recognition of the important role of surfaces in defect problems. Eshelby was the first to show that the surface contribution to the change in volume of the medium, when defects are present, is quite significant. When defects (internal stresses) are present in a bounded medium, one must invoke image forces such that the boundary of the medium is kept free of stresses (the equilibrium condition), analogous to the method of images in electrostatics. As in the latter case, the image stress in the defect problem should have no internal sources in the medium. A well-known result derived by this method is that the volume change of a finite medium when a single spherical point defect is present is $\gamma$ times the corresponding volume change in the infinite medium, where $\gamma=3(1-v) /(1+v)$ is the famous Eshelby factor and $v$ is Poisson's ratio. If $v$ is taken as $1 / 3$, then $\gamma$ is as large as $3 / 2$.

\section{The elastic dipole tensor}

Of central importance in the study of defects is the concept of the elastic dipole. Let a point force $f_{j}$ be acting at a point $\mathbf{r}^{\prime}$ in an elastic medium. The displacement $u_{j}$ at a point $\mathbf{r}$ caused by the force is

$$
u_{j}(\mathbf{r})=f_{1}\left(\mathbf{r}^{\prime}\right) G_{l j}\left(\left|\mathbf{r}-\mathbf{r}^{\prime}\right|\right) .
$$

Now suppose that in a small volume centred at $\mathbf{r}^{\prime}, N$ point forces $f_{k}^{\alpha}\left(\mathbf{r}^{\prime}+\mathbf{s}^{\alpha}\right)$ are acting at the points $\mathbf{r}^{\prime}+\mathbf{s}^{\alpha}(\alpha=1, \ldots, N)$. Then (14) must be generalised to

$$
\begin{aligned}
u_{l}(\mathbf{r}) & =\sum_{\alpha=1}^{N} f_{k}^{\alpha}\left(\mathbf{r}^{\prime}+\mathbf{s}^{\alpha}\right) G_{l k}\left(\left|\mathbf{r}-\mathbf{r}^{\prime}-\mathbf{s}^{\alpha}\right|\right) \\
& =\left[\sum_{\alpha=1}^{N} f_{k}^{\alpha}\right] G_{l k}\left(\left|\mathbf{r}-\mathbf{r}^{\prime}\right|\right)+\left[\sum_{\alpha=1}^{N} s_{j}^{\alpha} f_{k}^{\alpha}\right] G_{l k, j^{\prime}}\left(\left|\mathbf{r}-\mathbf{r}^{\prime}\right|\right) \\
& +\frac{1}{2}\left[\sum_{\alpha=1}^{N} s_{j}^{\alpha} s_{m}^{\alpha} f_{k}^{\alpha}\right] G_{l k, j^{\prime} m^{\prime}}\left(\left|\mathbf{r}-\mathbf{r}^{\prime}\right|\right) .
\end{aligned}
$$

Here the second equality is obtained by expanding the Green function in a Taylor series about $\left(\left|\mathbf{r}-\mathbf{r}^{\prime}\right|\right)$. (Note that $G_{l k, j^{\prime}}$ stands for $\left.\partial G_{l k} / \partial x_{j}^{\prime}\right)$. The first term in the expansion vanishes, because for physically realizable defects the invariance of the entire system under a rigid infinitesimal translation implies that

$$
\sum_{\alpha} f_{k}^{\alpha}\left(\mathbf{r}^{\prime}+\mathbf{s}^{\alpha}\right)=0 \text {. }
$$

The elastic dipole $\left(P_{j k}\right)$, tripole $\left(P_{j k l}\right)$, etc are then defined as the quantities in parentheses in the successive terms that remain:

$$
\begin{aligned}
& P_{j k}=\sum_{\alpha=1}^{N} s_{j}^{\alpha} f_{k}^{\alpha}, \\
& P_{j m k}=\sum_{\alpha=1}^{N} s_{j}^{\alpha} s_{m}^{\alpha} f_{k}^{\alpha}, \text { etc. }
\end{aligned}
$$

The displacement field due to a dipole is thus

$$
u_{l}(\mathbf{r})=P_{j k} G_{l k, j^{\prime}}\left(\left|\mathbf{r}-\mathbf{r}^{\prime}\right|\right) \text { and } P_{j k}=P_{k j} .
$$


It can be shown easily that a dipole is simulated by a body force according to

$$
f_{i}(r)=P_{i k} \delta_{, k}\left(r-r^{\prime}\right)
$$

The importance of the elastic dipole can be judged from the following result due to Kovács (1978): Any lattice defect can be described by a surface distribution of elastic dipoles. To establish this fact, consider a surface $S$ in a medium, and let it undergo a transformation such that the surface element $d S_{m}^{\prime}$ at a position $\mathbf{r}^{\prime}$ on the surface has a displacement $a_{l}\left(\mathbf{r}^{\prime}\right)$. Due to this transformation, the surface element $d S_{m}^{\prime}$ becomes a source of an elastic singularity with a dipole strength which can be defined by the expression

$$
\mathrm{d} P_{i k}=C_{i k l m} a_{l} \mathrm{~d} S_{m}^{\prime}
$$

The total strength of the defect is given by

$$
P_{i k}=\int_{S} C_{i k l m} a_{l} \mathrm{~d} S_{m}^{\prime}
$$

The displacement field of the defect is obtained by applying (18) and integrating over the dipole distribution

$$
\begin{aligned}
u_{n}(\mathbf{r}) & =\int G_{n i, k^{\prime}}\left(\mathbf{r}-\mathbf{r}^{\prime}\right) \mathrm{d} P_{i}\left(\mathbf{r}^{\prime}\right) \\
& =C_{i k l m} \int_{S} G_{n i, k^{\prime}}\left(\mathbf{r}-\mathbf{r}^{\prime}\right) a_{l}\left(\mathbf{r}^{\prime}\right) \mathrm{d} S_{m}^{\prime} .
\end{aligned}
$$

To apply this result to a dislocation, consider a cut in the medium over the surface $S_{C}$ bounded by a closed curve $C$. The positive direction of the normal to $S_{C}$ is related to $C$ by the right hand rule. Now let each point on $S_{C}$ be given a displacement $a_{l}\left(\mathbf{r}^{\prime}\right)=b_{l}$, b being a constant vector. Then we obtain the familiar Burgers (1939) result

$$
u_{n}(r)=C_{i k l m} b_{i} \int_{S_{C}} G_{l n, m}\left(r-r^{\prime}\right) \mathrm{d} S_{k}^{\prime},
$$

which is the displacement field of a dislocation loop $C$ of Burgers vector $\mathbf{b}$.

The displacement field of an MHI can be obtained by choosing for $S$ a closed surface enclosing a volume $V \neq 0$ and considering the displacement $a_{l}\left(r^{\prime}\right)$ inside and outside $S$ :

$$
a_{l}\left(\mathbf{r}^{\prime}\right)=\left\{\begin{array}{l}
u_{l}^{T}\left(r^{\prime}\right), \text { if } \mathbf{r}^{\prime} \in V \\
0, \text { otherwise }
\end{array}\right.
$$

Using (18) and applying Gauss's theorem, one obtains

$$
u_{n}(\mathbf{r})=\int_{V} C_{i k l m} u_{l, m}^{T} G_{n i, k^{\prime}} \mathrm{d} V^{\prime}+\int_{V} C_{i k l m} u_{l}^{T} G_{n i, k^{\prime} m^{\prime}} \mathrm{d} V^{\prime} .
$$

As the Green tensor is symmetric and satisfied the equation

$$
C_{i k l m} G_{n i, k^{\prime} m^{\prime}}=-\delta_{n l} \delta\left(\mathbf{r}-\mathbf{r}^{\prime}\right)
$$


we obtain

$$
u_{n}(\mathbf{r})= \begin{cases}\int_{V} \sigma_{i k}^{T} G_{n i, k^{\prime}} \mathrm{d} V^{\prime}-u_{n}^{T}, & \text { if } r \in V, \\ \int_{V} \sigma_{i k}^{T} G_{n i, k^{\prime}} \mathrm{d} V^{\prime}, & \text { if } r \notin V,\end{cases}
$$

where $\sigma_{i k}^{T}=C_{i k l m} u_{i, m}^{T}$. This result is essentially the same as that obtained by Eshelby (1961) if we identify $\frac{1}{2}\left(u_{l, m}^{T}+u_{m, l}^{T}\right)$ with the transformation strain $e_{m l}^{T}$. The total strength of this defect is

$$
\begin{aligned}
P_{i k} & =\oint_{S} C_{i k l m} u_{l}^{T} \mathrm{~d} S_{m}^{\prime} \\
& =\int_{V} C_{i k l m} u_{l, m}^{T} \quad \mathrm{~d} V^{\prime}=\int_{V} \sigma_{i k}^{T} \mathrm{~d} V^{\prime} .
\end{aligned}
$$

Thus an MHI can be interpreted as a space distribution of dipoles with a volume density $\sigma_{i k}^{T}$. A PFI can be treated similarly (Kovàcs 1978), and the results of Eshelby (1957) rederived.

The interaction energy $U$ between the defect, a strain field $e_{i k}^{A}$ and the associated force $F_{n}^{t}$ acting on the defect are expressed by the following general relations:

$$
\begin{aligned}
U & =-\int e_{i k}^{A}\left(\mathbf{r}^{\prime}\right) \mathrm{d} P_{i k}\left(\mathbf{r}^{\prime}\right) \\
& =-\int_{S} C_{i k l m} e_{i k}^{\prime}\left(\mathbf{r}^{\prime}\right) a_{l}\left(\mathbf{r}^{\prime}\right) \mathrm{d} S_{m}^{\prime} . \\
F_{n}^{t} & =\int e_{i k, n}^{A} \mathrm{~d} P_{i k} \\
& =C_{i k l m} \int_{S} e_{i k, n}^{A}\left(\mathbf{r}^{\prime}\right) a_{l}\left(\mathbf{r}^{\prime}\right) \mathrm{d} S_{m}^{\prime} .
\end{aligned}
$$

These equations are applicable to any type of defect. Thus, substituting in (30) the displacement appropriate for a dislocation and using Stokes' theorem, one recovers the Peach-Koehler (1950) expression for the force

$$
F_{n}^{\mathrm{disl}}=\oint_{C} \varepsilon_{m n k} \sigma_{l m}^{A} b_{l} \mathrm{~d} l_{k}^{\prime},
$$

where $\sigma_{l m}^{\prime}$ is the stress field associated with the strain $e_{l m}^{\prime}$ and $\mathrm{d} l_{k}^{\prime}$ is a line element along $C$.

\section{Generalizations of elastic continuum models}

An important step towards bridging the gap between the elastic continuum approach outlined in the previous sections and the more detailed atomistic calculations has been achieved on two different fronts: the anisotropic continuum approach and the nonlocal continuum approach. 


\subsection{The anisotropic continuum models}

These models are well described in the recent book by Teodosiu (1982) which also contains an exhaustive set of relevant references. A point defect in a crystal lattice is better represented by an elastic dipole tensor which is anisotropic, conforming to the symmetry of the actual defect site in the lattice, whereas the host lattice may be modelled as an elastically isotropic medium. We mention as an example the calculation of the interaction energy between two point defects with cubic symmetry $O_{h}$ by Siems (1968). He showed that two elastic dipoles do not interact, and that the principal singularity of the elastic interaction energy between two such defects is the dipoleoctupole interaction. Models along these lines have been proposed for faceted voids in irradiated crystals (Evans et al 1972; Malen and Bullough 1971).

Another approach is to consider spherically symmetric point defects in a host medium that is elastically anisotropic. The major drawback of this approach is the nonavailability of a closed form analytic expression for the Green tensor. For symmetries other than hexagonal symmetry (Kröner 1953), the solutions are in the form of series, thus necessitating perturbation-type calculations. For these reasons of mathematical inconvenience, not much work has been done along these lines. (For point defects, see Eshelby 1956; for line defects in an anisotropic host medium, Teodosiu 1982; Steeds and Willis 1979).

\subsection{The nonlocal elastic continuum models}

Eringen and Edelen (1972) and Eringen $(1972,1976)$ have developed a new theory of elasticity called nonlocal elasticity. This theory makes use of nonlocal elastic constants (a closer approximation to the force constants of the atomistic theories). The basic equations of linear isotropic elastic solids, for the static case and vanishing body forces are:

$$
\left.\begin{array}{rl}
\bar{\sigma}_{i k, k} & =0 \\
\bar{\sigma}_{i k} & =\int_{V} \sigma_{i k}^{N L}\left(\mathbf{r}, \mathbf{r}^{\prime}\right) \mathrm{d}^{3} r^{\prime}, \\
\sigma_{i k}^{N L} & =\alpha\left(\left|\mathbf{r}-\mathbf{r}^{\prime}\right|\right) C_{i k l m} e_{l m}^{\prime}, \\
e_{l m}^{\prime} & =\frac{1}{2}\left(u_{l, m^{\prime}}+u_{m, l^{\prime}}\right) .
\end{array}\right\}
$$

Here $\alpha(|\mathbf{R}|)$ attenuates rapidly as $R \rightarrow 0$, it is a continuous function which depends on a parameter $a$ such that when $a \rightarrow 0, \alpha(R) \rightarrow \delta(R)$ and the classical equations of elasticity are recovered. Here $e_{l m}^{\prime} \equiv e_{l m}\left(\mathbf{r}^{\prime}\right)$ and $u_{l, m^{\prime}}=\partial u_{l} / \partial x_{m}^{\prime}$. The stress $\sigma^{N L}(\mathbf{r})$ is the nonlocal stress in the sense that its value at $\mathbf{r}$ depends on the strain at all $\mathbf{r}^{\prime} \in V$. Eringen has shown that the first equation in (32) is satisfied if and only if $\left(C_{i k l m} e_{l m}\right)_{, k} \equiv \sigma_{i k, k}=0$ in $V$. This means that the displacement field remains the same in the nonlocal theory as in the classical theory. The stresses differ in the two theories.

Kovàcs and Vörös (1979) showed that the appropriate body force $F_{i}$ to be included in the generalization of $(32)$ is

$$
F_{i}(\mathbf{r})=\int \alpha\left(\mathbf{r}-\mathbf{r}^{\prime}\right) f_{i}\left(\mathbf{r}^{\prime}\right) \mathrm{d} V^{\prime},
$$

where $f_{j}(\mathbf{r})$ is the body force in the classical theory (see (1)). The force acting on an elastic 
dipole is then

$$
F_{j}(\mathbf{r})=\mathbf{P}_{j k}\left(\mathbf{r}^{\prime}\right) \alpha_{, k}\left(\mathbf{r}-\mathbf{r}^{\prime}\right)
$$

and the interaction energy $U$ between the elastic dipole and a displacement field $u_{i}^{A}$ is

$$
U(r)=-\int P_{j k}(\mathbf{r}) \alpha\left(\mathbf{r}^{\prime}-\mathbf{r}\right) \mathbf{e}_{j k}^{A}\left(\mathbf{r}^{\prime}\right) \mathrm{d} V^{\prime}
$$

Note that the interaction depends on the strain field at all points in the medium. The force acting on the dipole is

$$
f_{n}=\int P_{j k}(\mathbf{r}) \alpha\left(\mathbf{r}^{\prime}-\mathbf{r}\right) e_{j k, n}^{A}\left(\mathbf{r}^{\prime}\right) \mathrm{d} V^{\prime} .
$$

Note that (34)-(36) reduce to the corresponding equations of the classical theory, i.e. to (19), (29) and (30) when $\alpha\left(\mathbf{r}^{\prime}-\mathbf{r}\right) \rightarrow \delta\left(\mathbf{r}^{\prime}-\mathbf{r}\right)$.

Since any defect can be described as a surface distribution of dipoles (discussed in $\S 5$ ), it is interesting to generalise the results of the local theory for a surface distribution of dipoles. Kovács and Vörös (1979) have shown that (29) then generalises to the form

where

$$
U=-\int_{S} \bar{\sigma}_{l m}^{A}\left(\mathbf{r}^{\prime}\right) a_{l}\left(\mathbf{r}^{\prime}\right) \mathrm{d} S_{m}^{\prime}
$$

$$
\bar{\sigma}_{i k}(\mathbf{r})=\int_{V} \sigma_{i k}^{N L, A}\left(\mathbf{r}, \mathbf{r}^{\prime}\right) \mathrm{d} V^{\prime}=\int_{V} \alpha\left(\left|\mathbf{r}-\mathbf{r}^{\prime}\right|\right) C_{i k l m} e_{l m}^{A} \mathrm{~d} V^{\prime}
$$

and $a_{l}(\mathbf{r})$ and $S$ are as defined in $\S 5$. Similarly, (30) generalises to

$$
f_{n}=\int_{s} \bar{\sigma}_{l m}^{A}\left(\mathbf{r}^{\prime}\right) a_{l}\left(\mathbf{r}^{\prime}\right) \mathrm{d} S_{m}^{\prime} .
$$

It is easy to show that the self-energy of the defect is

$$
U_{s}=-\frac{1}{2} \int_{S} \bar{\sigma}_{l m}^{s}\left(\mathbf{r}^{\prime}\right) a_{l}\left(\mathbf{r}^{\prime}\right) \mathrm{d} S_{m}^{\prime},
$$

where $\tilde{\sigma}_{l m}^{s}$ is the stress field due to the defect alone.

As expected, the stress singularities and the embarassing infinite self-energies in the usual defect calculations are replaced by well-defined, finite quantities in the nonlocal theory. This is closer to physical reality, and the advantage of obtaining realistic defect properties without performing tedious atomistic calculations makes this approach very attractive. For lack of space, we quote a single result to substantiate this remark. The interaction energy $U$ between two dilation centres of volume increments $\Delta V_{1}$ and $\Delta V_{2}$ is given by

$$
U=2 \mu \frac{(1-v)}{(1-2 v)} \Delta V_{1} \Delta V_{2} \alpha(R)
$$

where $R$ is the distance between the two defects. (Note that when $\alpha(R)$ is replaced by $\delta(R), U \rightarrow 0$, since $R \neq 0$.) The self-energy $U_{s}$ of a dilation centre of volume increment $\Delta V$ is

$$
U_{s}=\frac{1}{2} P \Delta V \alpha(0)
$$


A convenient representation of the kernel $\alpha(r)$ is the Gaussian

$$
\alpha(R)=\frac{\pi^{3 / 2} k^{3}}{a^{3}} \exp \left(-k^{2} R^{2} / a^{2}\right)
$$

It is natural to interpret the parameter $a$ as the lattice parameter. The other parameter $k$ is estimated by Eringen $(1977 \mathrm{a}, \mathrm{b})$ from a consideration of the critical shear stress needed to create a dislocation. This leads to $k=0.94$ for fcc metals. In fcc metals $\Delta V=a^{3} \varepsilon / 4$ where $\varepsilon=\left(V-V_{0}\right) / V_{0}$, the relative volume difference between the host and the alloying atoms. Then, using $\nu=1 / 3$ and $k=0.94$, one obtains $U_{s}=0.56 \mu a^{3} \varepsilon^{2}$. This is to be compared with the atomistic model calculation of Flinn and Maradudin (1962) which yields $U_{s} \approx 0.54 \mu a^{3} \varepsilon^{2}$.

Other examples of the application of the nonlocal theory include expressions for the stress field, strain energy, etc. of screw and edge dislocations (Eringen 1977a, b; Eringen and Balta 1979), as well as a nonsingular expression (Eringen et al 1977) for the stressconcentration function in the problem of an elastic plate with a sharp crack and subjected to a uniform tension (at infinity) perpendicular to the crack (the Griffith problem). These results show the potential utility of the nonlocal elastic models of defects which certainly needs to be explored much more elaborately. Moreover, calculations in nonlocal elastic theory are much simpler than the corresponding ones in the anisotropic elasticity approach briefly described in $\$ 6.1$.

\section{The linear continuum theory of dislocations}

Electron microscopic observations show that deformed crystals have numerous dislocation lines which are very often randomly tangled. A convenient way to describe these dislocations is via the dislocation density tensor $\alpha_{i j}(\mathbf{r})$ (Nye 1953). Consider an arbitrary point $P$ in the material. In the neighbourhood of $P$, within a small area element $\mathrm{d} \mathbf{S}$, there are several dislocation lines with Burgers vector $\mathbf{b}^{(a)}(a=1,2 \ldots n)$ in the directions $\hat{v}^{(a)}$, as shown in figure 3. The effective Burgers vector $\mathrm{db}$ of $n$ dislocations threading the surface $\mathrm{d} S$ is then given by

$$
\mathrm{d} S_{i} \alpha_{i j}=\sum_{a=1}^{n}\left(\hat{v}^{(a)} . \hat{v}\right) b_{j}^{(a)} \equiv \mathrm{d} b_{j}
$$

where $\hat{v}$ is the unit vector at $P$ defining the element $\mathrm{dS}$. The scalar product $\left(\hat{v}^{a}, \hat{v}\right)$ measures the threading of the surface $\mathrm{d} S$ by the $a$ th dislocation. In integral form, (44) is written as

$$
\int_{S} \mathrm{~d} S_{i} \alpha_{i j}=b_{j}=\oint_{C} \mathrm{~d} u_{j},
$$

where $C$ is the closed curve bounding the surface $S$. (Kröner 1964 and Noll 1967 have

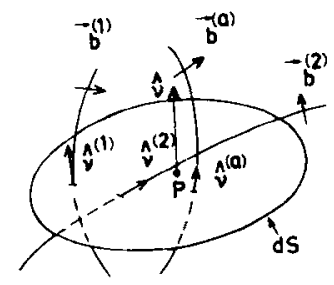

Figure 3. The area element $d \mathbf{S}$ showing the threading of dislocation lines. 
defined $\alpha_{i j}$ somewhat differently; see Teodosiu (1982)). Now

$$
\left.\alpha_{i j}=\varepsilon_{i k l} \partial_{k} \beta_{l j} \quad \text { (or } \alpha=\operatorname{curl} \beta\right),
$$

where $\beta_{i j}(\mathbf{r})=u_{j, i}(\mathbf{r})$ is the gradient of the elastic displacement. Using (46) in (45) and invoking Stokes theorem, we find

$$
\partial_{i} \alpha_{i j}=\alpha_{i j, i}=0,(\text { or } \operatorname{div} \alpha=0 \text { ), }
$$

because the divergence of a curl vanishes. Physically this relation means that dislocations cannot end inside the medium. Another feature of the $\alpha$-tensor is worth noting. The first index $i$ of $\alpha_{i j}$ denotes the average direction of the dislocation lines whereas the second index $j$ denotes the direction of the Burgers vector. Thus the diagonal components of $\alpha$ correspond to screw dislocations and the off-diagonal elements to edge dislocations. Since the diagonal and nondiagonal components of a tensor are not invariant notions, the classification of dislocations into edge and screw types is not to be taken too seriously.

The introduction of the $\alpha$-tensor has given impetus to the development of the beautiful differential-geometric theory (see $\S 9$ ). The connection with geometry arises from the physical fact that dislocations involve a certain curvature or bending of the lattice network, thus imparting a curvature to the continuum (Nye 1953). To be more precise, the rotation tensor $\omega_{i j}$ defined as the antisymmetric part of $\beta$ leads to an axial rotation vector $\omega_{k}=\frac{1}{2} \varepsilon_{i j k} \omega_{i j}$. The difference in $\omega$ between two points separated by an infinitesimal distance $d \mathbf{r}$ is given by

where

$$
\mathrm{d} \omega_{j}=\left(\varepsilon_{j k l} \hat{l}_{k} e_{l i}+\kappa_{i j}\right) \mathrm{d} x_{i}
$$

$$
\kappa_{i j}=\alpha_{i j}-\frac{1}{2} \alpha_{k k} \delta_{i j}
$$

is the Nye's curvature tensor (see also Kröner 1981). Thus, in the absence of elastic strains, the presence of a continuous distribution of dislocations alone leads to a rotation $d \theta$ between two points separated by a distance $d \mathbf{r}$ given by $d \theta_{j}=\kappa_{i j} \mathrm{~d} x_{i}$. It is also straightforward to calculate the incompatibility tensor $\eta$ associated with the presence of the dislocation distribution. We quote the final result (Kröner 1981):

$$
\begin{gathered}
\eta_{i j}=-\varepsilon_{i k m} \varepsilon_{j l n} \partial_{k l} e_{m n}=\varepsilon_{i k m} \partial_{k} \kappa_{m j}=-\frac{1}{2}\left(\varepsilon_{i l n} \partial_{n} \alpha_{j l}\right)_{(i j)}, \\
\bar{\eta}=\text { inc } e=\operatorname{curl} \boldsymbol{\kappa}=(\alpha \times \nabla)_{s y m}=-\operatorname{curl} \alpha^{T} .
\end{gathered}
$$

It is evident that the divergence of $\eta$ vanishes.

\section{The internal stress problem and Kröner's theory}

Defects are characterized by the incompatibility tensor $\eta$ associated with them. For a continuous distribution of dislocations it is given by (49). A single dislocation is described as a limiting case of the continuous distribution with the help of delta functions:

$$
\alpha_{i j}=t_{i} b_{j} \delta(p) \delta(q)
$$

where $t_{i}$ is the tangent to the dislocation line and $p, q$ are suitable coordinates perpendicular to the dislocation line. For a spherical interstitial atom occupying a 
volume $\Delta V$ and located at $\mathbf{r}_{0}$, the incompatibility tensor is

$$
\eta=\Delta V(\vec{\nabla} \times I \times \stackrel{\leftarrow}{\nabla}) \delta\left(\mathbf{r}-\mathbf{r}_{0}\right)
$$

where $I$ is the unii tensor $\left(\delta_{i j}\right)$.

The main problem in defect theory is: given the tensor $\eta$ as a function of $r$, to calculate the internal stress and strain in the body. This is a boundary value problem for the equations $\partial_{i} \sigma_{i j}=0$ (force equilibrium condition in the bulk) and $\sigma_{i j} n_{j}=0$ on the surface $S$ of the body (the traction boundary condition) together with the incompatibility equation inc $\boldsymbol{e}=\boldsymbol{\eta}$. There are three ways of calculation of $\boldsymbol{e}$.

(i) The Eshelby-Eddington method: Eshelby (1956) uses the fact that, because of the tensor identities

$$
\operatorname{inc}(\operatorname{def} u)=0 \text { and } \operatorname{div}(\operatorname{inc} A)=0,
$$

any solution of the incompatibility equation can be written as

$$
\boldsymbol{e}=\operatorname{def} \mathbf{u}+\tilde{\boldsymbol{e}},
$$

where $\dot{e}$ is a particular solution of the equation and $\mathbf{u}$ is an arbitrary vector field. If we put $\eta=0$ on the boundary $S$, then following the method of Eddington in the general theory of relativity, one can write

$$
\check{e}(\mathbf{r})=\frac{1}{4 \pi} \int_{V} d^{3} \mathbf{r}^{\prime} \frac{\eta\left(\mathbf{r}^{\prime}\right)-(\operatorname{Tr} \eta) I}{\left|\mathbf{r}-\mathbf{r}^{\prime}\right|} .
$$

Next, by substituting (53) into the other field equation and the boundary conditions, one obtains a traction boundary-value problem of classical elasticity theory where $\mathbf{u}$ plays the role of a displacement field, while the body forces and the surface forces are given by

$$
\begin{aligned}
f_{k} & =C_{k l m n} \tilde{e}_{m n n, l}, \\
t_{k} & =-C_{k l m n} \tilde{e}_{m n} n_{l} .
\end{aligned}
$$

(ii) Mura's method: This method make use of the elegant Green function technique. The working relation (derived in Mura 1968; Teodosiu 1982) for the calculation of the elastic deformation tensor is

$$
\beta_{m r}(\mathbf{r})=\int \mathrm{d}^{3} r^{\prime} C_{p l q n} \varepsilon_{n r i} \alpha_{q t . l^{\prime}}\left(\mathbf{r}^{\prime}\right) G_{m p}\left(\left|\mathbf{r}-\mathbf{r}^{\prime}\right|\right)
$$

(iii) Kröner's method: Kröner (1981) exploited the analogy of the elastic field with the magnetostatic field. The following is the correspondence between quantities and relations in both theories. (See Seeger 1961 for an excellent review where reference to Kröner's earlier work are given.)

\begin{tabular}{ll} 
Elasticity & Magnetism \\
\hline vector quantity & scalar quantity \\
rank two tensor & vector \\
rank four tensor & rank two tensor \\
div & div \\
inc & curl
\end{tabular}




\begin{tabular}{ll} 
Elasticity & Magnetism \\
\hline div inc $\equiv 0$ & div curl $\equiv 0$ \\
def & grad \\
inc def $\equiv 0$ & curl grad $\equiv 0$ \\
Burgers vector $\mathbf{b}$ & current $\mathbf{I}$ \\
incompatibility tensor $\eta$ & current density $\mathbf{J}$ \\
strain tensor $e$ & magnetic intensity $\mathbf{H}$ \\
stress tensor $\sigma$ & magnetic induction $\mathbf{B}$ \\
elastic constant $\mathbf{C}$ & permeability $\mu$ \\
$\sigma=C: e$ & $\mathbf{B}=\mu \mathbf{H}$ \\
div $\sigma=0$ & div $\mathbf{B}=0$ \\
inc $e=\eta$ & curl $\mathbf{H}=\frac{4 \pi}{c} \mathbf{J}$ \\
tensor potential $\chi$ & vector potential $\mathbf{A}$ \\
$\sigma=$ inc $\chi$ & $\mathbf{B}=$ curl $\mathbf{A}$ \\
\hline
\end{tabular}

Another auxiliary tensor potential $\chi^{\prime}$ is introduced ingeniously by Kröner. This is given by

$$
\chi_{i j}^{\prime}=\frac{1}{2 \mu}\left(\chi_{i j}-\frac{v}{1+2 v} \chi_{k k} \delta_{i j}\right),
$$

the inverse relation being

$$
\chi_{i j}=2 \mu\left(\chi_{i j}^{\prime}+\frac{v}{1-v} \chi_{k k}^{\prime} \delta_{i j}\right) .
$$

$\chi^{\prime}$ satisfies the equations

$$
\nabla^{4} \chi^{\prime}=\eta \text { and } \operatorname{div} \chi^{\prime}=0 .
$$

Assuming that $\eta \rightarrow 0$ at the boundary of the infinite medium one calculates $\chi^{\prime}$ from the relation

$$
\chi_{i j}^{\prime}(\mathbf{r})=-\frac{1}{8 \pi} \int \mathrm{d}^{s} \mathbf{r}^{\prime}\left|\mathbf{r}-\mathbf{r}^{\prime}\right| \eta_{i j}\left(\mathbf{r}^{\prime}\right) .
$$

In principle this solves the internal stress problem, since $\sigma$ can now be calculated using $\sigma=\operatorname{inc} \chi$.

Kröner has also put the elastic theory of point defects in a broader perspective. According to him, the elastostatics of a continuum with extrinsic point defects (foreign interstitials) is analogous to electrostatics, whereas in intrinsic defects (vacancies, selfinterstitials) the elasticity theory is analogous to magnetostatics:

Electrostatics

Elastostatics with extrinsic defects

\begin{tabular}{ll}
\hline $\operatorname{div} \mathbf{D}=\rho$ & $\operatorname{div} \boldsymbol{\sigma}=-\mathbf{f}$ \\
$\operatorname{curl} \mathbf{E}=0$ & inc $e=0$ \\
$\mathbf{D}=\boldsymbol{\varepsilon} \cdot \mathbf{E}$ & $\sigma=C: \boldsymbol{e}$ \\
$U=\frac{1}{2} \mathbf{D} \cdot \mathbf{E}$ & $U=\frac{1}{2} \sigma \cdot \boldsymbol{e}$
\end{tabular}


Magnetostatics

Elastostatics with intrinsic defects (internal sources)

$\begin{array}{ll}\operatorname{div} \mathbf{B}=\mathbf{0} & \operatorname{div} \sigma=0 \\ \operatorname{curl} \mathbf{H}=\mathbf{J} & \text { inc } e=\eta \\ \mathbf{B}=\boldsymbol{\mu} \cdot \mathbf{H} & \sigma=C: \boldsymbol{e} \\ U=\frac{1}{2} \mathbf{B} \cdot \mathbf{H} & U=\frac{1}{2} \sigma: \boldsymbol{e}\end{array}$

The distinction between extrinsic and intrinsic defects is considered to be a most fundamental one, since this has deeper implications in terms of differential geometry. The terms 'diaelasticity' and 'paraelasticity' now acquire meanings by analogy with electro- and magnetostatics. Carbon atoms in iron are permanent elastic dipoles giving rise to paraelasticity; whereas, in diaelasticity, the induced elastic dipoles $p_{i j}^{\text {ind }}$ arise only in the presence of an applied elastic strain $e_{k i}$, according to $p^{\text {ind }}=\alpha: e$. The PFI leads to diaelasticity, while MFI leads to both para- and diaelastic effects. A quantitative measure of the para- or diaelasticity associated with a point defect is the corresponding polarizability. The calculation of this quantity is an important problem in the study of point defect relaxation as in the Snoek effect (see, e.g., Leibfried and Breur 1978). There are also ferroelastic solids possessing permanent macroscopic elastic dipole moment along favoured directions (see, e.g. Wadhawan 1982).

\section{Differential geometry of defects}

The description of defects in the foregoing was confined to linearalized theories. When finite deformations are included, the language of differential geometry and tensor calculus becomes indispensable. A summary of these theories is given by Kröner (1981) and we follow his presentation. The nature of the differential geometry is decided by the degrees of freedom associated with the atoms or molecules of the crystal. For constituent particles of the same kind, each having only three translational degrees of freedom (a Bravais lattice), affine geometry seems to be appropriate; whereas for a nonBravais lattice of molecules possessing rotational degrees of freedom in addition to translational ones, Finsler's geometry (Amari 1962) is required.

Consider for simplicity a cubic primitive Bravais lattice and choose the lattice lines as the Cartesian coordinate lines. The Cartesian coordinates of this defect-free, reference crystal are denoted by $x^{k}$. Let the crystal be deformed and let this configuration have coordinates $z^{k}$. The connection between infinitesimal increments in the coordinates in the two configurations is given by

$$
\mathrm{d} z^{k}=B_{m}{ }^{k} \mathrm{~d} x^{m}, B_{m}{ }^{k}=\partial z^{k} / \partial x^{m} .
$$

Now $\mathrm{d} x^{m}$ being the corresponding change in the undeformed configuration, the relative displacement is given by

$$
\mathrm{d} u^{k}=\left(B_{m}{ }^{k}-\delta_{m}{ }^{k}\right) \mathrm{d} x^{m}=\beta_{m}{ }^{k} \mathrm{~d} x^{m} .
$$

With the coordinates $x^{k}$ we associate an 'external observer' (also called the Eulerian frame of reference). Consider another observer, the 'internal observer' (the Lagrangian frame of reference) whose reference coordinates are 'dragged along' in the process of deformation. As long as the crystal is defect-free and the deformation is compatible, the internal observer feels no deformation. Let the coordinates of this frame of reference be 
$y^{k^{\prime}}$. Then the square of the distance between two infinitesimally separated points

$$
\mathrm{d} s^{2}=\delta_{k^{\prime} l^{\prime}} \mathrm{d} y^{k^{\prime}} \mathrm{d} y^{\prime \prime}
$$

is an invariant for this observer. In contrast, for the external observer, there is a change in $d s^{2}$ after a deformation takes place, given by

$$
\mathrm{d} s^{2}-\mathrm{d} s_{0}^{2}=\left(g_{k l}-\delta_{k l}\right) \mathrm{d} x^{k} \mathrm{~d} x^{l} \equiv 2 e_{k l} \mathrm{~d} x^{k} \mathrm{~d} x^{l} .
$$

Here $\mathrm{d} s_{0}^{2}$ is the value of $\mathrm{d} s^{2}$ before deformation, and

$$
g_{k l}=\delta_{m n} B_{k}{ }^{m} B_{l}{ }^{n} .
$$

The strain tensor can be easily shown to be

$$
e_{k l}=\frac{1}{2}\left(\beta_{k l}+\beta_{l k}\right)+\frac{1}{2} \beta_{k}{ }^{m} \beta_{l}{ }^{n} \delta_{m n} .
$$

Next, let us establish a connection between dislocations and torsion. Cartan (1928) introduced the concept of 'torsion' in a purely mathematical context. Later, Frank (1951) constructed the Burgers circuit to define a dislocation. That there is a remarkable similarity between Cartan's procedure for introducing torsion in differential geometry and Frank's construction for a dislocation was noted by Kondo $(1952,1955)$, Bilby et al (1955) and Bilby (1960). Frank considers the nonclosed path $A^{\prime} B^{\prime} C^{\prime} D^{\prime} E^{\prime}$ (see figure 4) around a dislocation in a crystal counting the steps (each step consists of one atomic spacing). The closer failure $\mathbf{A}^{\prime} \mathbf{E}^{\prime}$ is the Burgers vector. Cartan transports a vector $\mathbf{C D}$ along the path $\mathrm{CB}$ from $C$ to $B$ and then a vector $\mathrm{CB}$ along the path from $C$ to $D$. The vectors $C D=d_{1} \times(C), B A=d_{1} \times(B), C B=d_{2} x(C)$ and $D E=d_{2} \times(D)$ are all of infinitesimal length. The law of parallel transport of $d_{1} x(C)$ along $d_{2} x(C)$ can be written as

$$
\mathrm{d}_{1} x^{h}(B)=\mathrm{d}_{1} x^{h}(C)-\Gamma_{m l}{ }^{h} \mathrm{~d}_{1} x^{l}(C) \mathrm{d}_{2} x^{m}(C),
$$

where $\Gamma_{m l}{ }^{h}$ is the affine connection. Similar transport of $d_{2} x(C)$ along $d_{1} x(C)$ gives

$$
\mathrm{d}_{2} x^{h}(D)=\mathrm{d}_{2} x^{h}(C)-\Gamma_{m l}{ }^{h} \mathrm{~d}_{2} x^{l}(C) \mathrm{d}_{1} x^{m}(C) .
$$

The closure failure is thus

$$
\begin{aligned}
(\mathbf{A E})^{h} & =\mathrm{d}_{1} x^{h}(C)+\mathrm{d}_{2} x^{h}(D)-\mathrm{d}_{2} x^{h}(C)-\mathrm{d}_{1} x^{h}(B) \\
& =\Gamma_{m l}{ }^{h}\left(\mathrm{~d}_{1} x^{l} \mathrm{~d}_{2} x^{m}-\mathrm{d}_{2} x^{l} \mathrm{~d}_{1} x^{m}\right) \\
& =-\Gamma_{m l}{ }^{h} \mathrm{~d} S^{m l},
\end{aligned}
$$

where $\mathrm{d} S^{m l}=\left(\mathrm{d}_{1} x^{m} \mathrm{~d}_{2} x^{l}-\mathrm{d}_{1} x^{l} \mathrm{~d}_{2} x^{m}\right)$ is the area element enclosed by the four infinitesimal vectors. The tensor $\mathrm{d} S^{m l}$ is related to the area vector $\mathrm{d} S_{n}$ by the relation

$$
\mathrm{d} S^{m l}=\bar{\varepsilon}^{n m l} \mathrm{~d} S_{n}, \mathrm{~d} S_{n}=\frac{1}{2} \bar{\varepsilon}_{n m l} \mathrm{~d} S^{m l},
$$

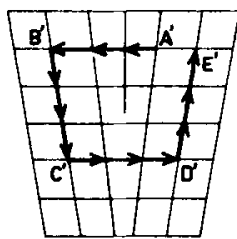

(a)

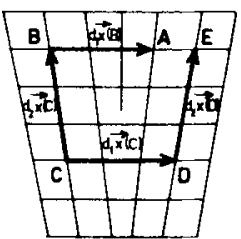

(b)

Figure 4. (a) Frank's circuit, (b) Cartan's circuit. 
where $\bar{\varepsilon}^{n m l}=g \bar{\varepsilon}^{n m l}$, with $g=\left(\operatorname{det}\left(g_{l m}\right)\right)^{1 / 2}$ (i.e., $\bar{\varepsilon}^{n m l}$ is the contravariant Levi-Civita tensor, etc.). Note that only the antisymmetric part of the connection contributes to the vector $(\mathbf{A E})^{h}$ in (70). This is Cartan's torsion tensor,

$$
T_{m l}{ }^{h}=\frac{1}{2}\left(\Gamma_{m i}{ }^{h}-\Gamma_{l m}{ }^{h}\right)
$$

Now, to establish contact with the dislocation density tensor $\alpha_{i j}$ defined in $\S 7$, note that by definition the Burgers elemental vector is

where

$$
\mathrm{d} b^{k}=\alpha^{n k} \mathrm{~d} S_{n} \equiv \alpha_{m l}{ }^{k} \mathrm{~d} S^{m l},
$$

$$
\alpha_{m l}{ }^{k}=\frac{1}{2} \bar{\varepsilon}_{n m l} \alpha^{n k}, \alpha^{n k}=\bar{\varepsilon}^{n m l} \alpha_{m l}{ }^{k} .
$$

On comparing $\mathrm{d} b^{k}$ with $(\mathbf{A E})^{k}$ we get

$$
T_{m l}^{k}=-\alpha_{m l}{ }^{k} .
$$

This basic identification of the dislocation density tensor with the torsion tensor is the starting point of the differential geometric approach.

The presence of torsion leads to a non-Riemannian geometry. It is known (Schouten 1954) that if the metric is to satisfy the relation $\nabla_{m} g_{l k}=0$ where $\nabla_{m}$ is the symbol for covariant derivative, then the most general form of the affine connection possible is related to the torsion as follows: writing $\Gamma_{m l}{ }^{h}=g^{h k} \Gamma_{m l k}$, it turns out that

where

$$
\Gamma_{m l k}=g_{m i k}+h_{m l k},
$$

and

$$
g_{m l k} \equiv \frac{1}{2}\left(\partial_{m} g_{k l}+\partial_{l} g_{m k}-\partial_{k} g_{l m}\right)
$$

$$
h_{m l k} \equiv T_{m l k}+T_{k m l}-T_{l k m} \text {. }
$$

Here $g_{m l k}$ is the Christoffel symbol of the second kind, and $h_{m l k}$ is the contortion tensor. The earlier definitions of the distortion tensor $\beta_{i k}$, the strain tensor $e_{i k}$ and the rotation tensor $\omega_{i k}$ are generalised to read

$$
\left.\begin{array}{rl}
\mathrm{d} \beta_{l k} & =\Gamma_{m l k} \mathrm{~d} x^{m}, \\
\mathrm{~d} e_{l k} & =\Gamma_{m(l k)} \mathrm{d} x^{m}, \\
\mathrm{~d} \omega_{l k} & =\Gamma_{m[l k]} \mathrm{d} x^{m},
\end{array}\right\}
$$

where $(l k)$ and $[l k]$ respectively stand for symmetric and antisymmetric combinations of these indices. Equation (9) of the linearized theory, which can also be written as

$$
\vec{\nabla} \times e \times \stackrel{\nabla}{\nabla}-\operatorname{sym}\{\alpha \times \stackrel{\leftarrow}{\nabla}\}=0
$$

is generalised in the nonlinear theory to the vanishing of the curvature tensor, i.e.,

$$
R_{\text {nmlk }}=0 \text {, }
$$

where $R_{n m l k}=g_{k h} R_{n m l}^{h}$ and

$$
R_{n m l}{ }^{h}=2\left(\partial_{n} \Gamma_{m l}{ }^{h}-\Gamma_{m p}{ }^{h} \Gamma_{n l}{ }^{p}\right)[n m] .
$$

Equation (81) is the second basic law of the differential geometric theory of lattice defects. Physically, this means that if a crystal has dislocations as the only defects in it during deformation, and if it does not develop cracks, then the dislocation distribution must be accompanied by elastic strains such that the curvature tensor vanishes. 
The second basic identification states that elementary extrinsic defects (extra matter on interstitial sites, fission fragments, $\alpha$-particles stuck in the medium, etc) cause a rotation-like curvature $\left(R_{n m[k]} \neq 0, R_{n m(l k)}=0\right)$. According to the third basic identifcation, intrinsic point defects like self-interstitials and vacancies lead to a non-metric curvature $\left(R_{n m[l k]}=0, R_{n m(l k)} \neq 0\right)$. The non-metric situation is not as well understood in differential geometry as the metric connection. When extrinsic defects are present in addition to dislocations, a beautiful analogy emerges between the field equations of defects and Einstein's equations in the general theory of relativity. To see this, we note that the curvature tensor $R_{n m l k}$ is antisymmetric in $l$ and $k$ for the metric geometry, and, by definition, it is also antisymmetric in $n$ and $m$. Thus, without any loss of information, it can be replaced by a second rank tensor $E^{i j}$ (called the Einstein tensor) given by

$$
E^{i j}=\frac{1}{4} \bar{\varepsilon}^{i n m} \bar{\varepsilon}^{j l k} R_{n m l k} .
$$

It can be shown that, in the linear approximation, (83) reduces to

$$
E=- \text { inc } e+\operatorname{curl} \boldsymbol{\kappa} .
$$

Note that when $R_{n m l k}=0, E^{i j}=0$. When extra matter is present, the Einstein tensor does not vanish and becomes equal to the matter tensor $M^{i j}$ or $E=M$. This is analogous to the Einstein's field equation. Thus the defects in a crystal are analogous to matter in the universe. "Without matter, the universe is not interesting; without defects, a crystal is dead--" (Kröner 1981).

The approach to defect theories via differential geometry is undoubtedly an elegant one. There is a related approach by Wang (1967) and Noll (1967) which starts with the philosophy that the constitutive law of the continuum being fundamental, it must determine the geometry. There are attempts at constructing a Yang-Mills type of minimal coupling theory for materials with dislocations and disclinations (Kadić and Edelen 1982; Edelen 1980). The work of Julia and Toulouse (1979) and that of Dzyaloshinskii and Volovik (1978) are efforts in the same direction. There are further treatments of the differential geometry of defects by Gairola (1979), Zorawski (1967) and Marcinkowski (1979). Marcinkowski in several publications has applied differential geometric techniques extensively to dislocations on surfaces, grain boundaries, cracks, etc.

In spite of its mathematical elegance, the differential-geometric approach to defect problems has not helped much in the development of the phenomenological theory of plasticity. The reason is that, in contrast to the case of electric or magnetic lines of force in electromagnetic theory, dislocation lines almost always form very irregular interconnected networks. This is the main reason why the continuum theory of dislocations and the differential-geometric approach as such can never constitute a good macroscopic theory of plasticity. Dislocations piercing through an area element in the crystal usually appear with positive as well as negative signs so that their resultant Burgers vector is often zero or has a very small value. This results in a very small magnitude for the dislocation density $\alpha$. To understand this, consider for example the configuration of dislocations shown in figure 5. All the dislocation lines are in the $z$ direction; positive $(\mathrm{L})$ and negative $(T)$ dislocations have Burgers vectors $+b$ and $-b$, respectively. Let the domain be divided by a mesh as shown in the figure and let $a^{2}$ be the area of each small square. If we consider as the area $\Delta S_{3}$ the rectangle $\mathrm{AA}^{\prime} \mathrm{B}^{\prime} \mathrm{B}$, $\alpha_{31}=-b / a^{2}$; if $\Delta S_{3}$ is the rectangle $\mathrm{BB}^{\prime} \mathrm{C}^{\prime} \mathrm{C}, \alpha_{31}=+b / a^{2}$; whereas if area $\Delta S_{3}$ $=\mathrm{AA}^{\prime} \mathrm{C}^{\prime} \mathrm{C}, \alpha_{31}=0$. Note that if we let $\Delta S_{3} \rightarrow 0$ around the point $P_{1}$, then $\alpha_{31}(P)=$ $\pm b_{1} \delta\left(P-P_{1}\right)$, where the + sign is applicable if there is a positive dislocation at $P_{1}$, and 


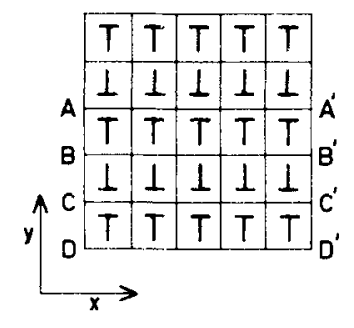

Figure 5. Positive and negative edge dislocations arrays piercing through a rectangular region.

the - sign if there is a negative dislocation. This means that the microscopic theory of dislocations is built into the formalism, but that in the process of coarse-graining over the distribution of dislocations, too much information seems to be lost.

Kröner (1970) has suggested the following way to rectify this deficiency. According to him, the random distribution of dislocations calls for the use of statistical mechanics. Information about the actual dislocation distribution may be given by $n$-point correlation tensor functions $\left\langle\alpha\left(\mathbf{r}_{1}\right) \alpha\left(\mathbf{r}_{2}\right) \ldots \alpha\left(\mathbf{r}_{n}\right)\right\rangle$ which give the probability of finding dislocation elements at $\mathbf{r}_{1}, \mathbf{r}_{2} \ldots \mathbf{r}_{n}$. Thus one considers, instead of a single real specimen, a whole ensemble of crystals all filled with dislocation line elements in such a way that the ensemble represents all possible realizations of a given macroscopic dislocation state. One may impose extra conditions by prescribing the total energy, the total dislocation length, etc. These would correspond to various types of ensembles (analogous to the well-known microcanonical, canonical and grand canonical ensembles). Kröner (1970) shows that the information contained in the two-point correlation $\left\langle\alpha_{i j}(\mathbf{r}) \alpha_{k l}\left(r^{\prime}\right)\right\rangle$ is quite extensive. For example, it gives information about the total dislocation length in a given volume element, the density of dislocation dipoles, etc. It is (obviously) suitable for describing planar dislocation networks, and, to some extent, dislocation pileups as well. Kröner and Teodosiu (1974) have made the important observation that the internal mechanical state, instead of being represented by the dislocation density tensor and its higher-order correlations, can also be specified by means of the fluctuations of the stress and elastic strain produced by the dislocations. This is of interest because it is the fluctuating stresses and strains that determine the deformation behaviour of materials. This line of approach is very attractive, but much work needs to be done. For example, if the dislocation elements are the basic entities of the system which we want to describe by statistical mechanics, what is the counterpart of Liouville's theorem that would guarantee that the known equations of motion of the dislocation line elements are satisfied? It is worth mentioning here that in another attempt to rectify the shortcomings of the dislocation density tensor, Kroupa (1962) has introduced the dislocation loop density as an additional variable. This approach too has not yet had any practical application.

\section{Continuum mechanics of moving dislocations}

Kosevich (1979) has generalised the basic equations of continuum mechanics taking into account the motion of dislocations during deformation. He has given a very simplified treatment under the (rather drastic) assumption that the energy conservation equation remains unchanged. (This is equivalent to assuming that no heat is generated in the process of dislocation motion). Thus, only the equations of continuity expressing the (local) conservation of mass and (linear) momentum are considered. One begins 
with the fundamental equation which introduces dislocations into elasticity theory, namely, (46). If dislocations remain static during the deformaton, then the velocity field $V_{k}(\mathbf{r})$ is related to the elastic distortion field $\beta_{i k}(\mathbf{r})$ by $\partial_{i} V_{k}=\partial_{t} \beta_{i k}$. However, when dislocations move, this equation must be generalized to

$$
\partial_{i} V_{k}=\partial_{t} \beta_{i k}-j_{i k}
$$

Here $j_{i k}(\mathbf{r})$ is the dislocation flux tensor which must be so chosen that (85) is consistent with (46). Taking the curl of (85) and using (46), one obtains the (local) law of conservation for the Burgers vector

$$
\partial_{t} \alpha_{i k}+\varepsilon_{i l m} \partial_{l} j_{m k}=0 \text {. }
$$

If $C$ is a closed curve bounding a surface $S$, then (86) can be expressed in the alternate form

$$
\mathrm{d} b_{k} / \mathrm{d} t=-\oint_{C} j_{i k} \mathrm{~d} x_{i} .
$$

The total distortion $\beta_{i k}^{T}$ is the sum of the elastic distortion $\beta_{i k}$ and the plastic distortion $\beta_{i k}^{P}$. Since $\partial_{i} V_{k}=\partial_{t} \beta_{i k}^{T}$ by definition, we have evidently $\partial_{t} \beta_{i k}=-j_{i k}$. Hence

$$
\partial_{t} e_{i k}^{p}=-j_{(i k)}
$$

which is the Orowan equation. For a continuous distribution of dislocations,

$$
j_{i k}=\varepsilon_{i l m} \sum_{a} b_{k} V_{m}\left(\mathbf{r}, \hat{v}^{(a)}\right) v_{l}^{(a)}
$$

(compare with (46) which defines $\alpha_{i k}$ ). The trace $j_{k k}$ of the flux tensor $j_{i k}$ is of special interest in connection with the continuity equation for mass. Noting that the relative change in density $\delta \rho$ is given by $e_{k k}=-\delta \rho / \rho$ (where $\rho$ is the density of the medium) and using (85), one obtains

$$
\partial_{t} \rho+\operatorname{div}(\rho \mathbf{V})=-\rho j_{k k} \text {. }
$$

The 'complete system' of dynamical equations can then be presented in the form

$$
\left.\begin{array}{l}
\partial_{i} \sigma_{i k}=\rho \partial_{t} V_{k} \quad \text { (Newton's Law), } \\
\sigma_{i k}=C_{i k l m} \beta_{l m} \quad \text { (elastic constitutive Law), } \\
\varepsilon_{i l m} \partial_{l} \beta_{m k}=-\alpha_{i k}, \\
\partial_{i} V_{k}=\partial_{t} \beta_{i k}-j_{i k},
\end{array}\right\}
$$

where the tensors $\alpha_{i k}$ and $j_{l k}$ must be specified. Note that $\alpha_{i k}$ and $j_{l k}$ must satisfy (47) and (86).

We mention here that a lattice defect approach to plasticity and viscoplasticity has been proposed by Kröner and Teodosiu (1974), in which particular emphasis is laid on dislocations. These authors propose a complete set of equations which consist of the continuity equations including the law of conservation of energy, and the thermoelastic constitutive equations for the stress and the heat flux. However, the theory is rather formal and has not yet been tested on any practical problem.

\section{The quantum field theory approach}

Field theorists have recently been interested in defects in crystals. Defects (termed 'extended objects' to stress the generality of the formalism) are viewed as classical 
macroscopic objects in a quantum ordered state (a perfect crystal). They are a macroscopic manifestation of the local condensation of bosons associated with a quantum mechanical collective mode (the phonons). There is an efficient tool called the boson transformation technique which describes the condensation process mathematically. In high energy physics, the topological structure of extended objects has been of great interest, being connected with the problems of quark confinement and particle structure. The boson transformation approach to defects was motivated by the success of the theory of solitorıs. A soliton is a localized wave solution of a classical nonlinear equation which behaves like a particle. The search for soliton solutions in three space dimensions is a challenging problem in itself. Their localized nature suggests that dislocations, point defects, grain boundaries, etc. are somehow related to soliton-like objects in three-dimensions. As it would require a rather lengthy presentation of the field-theoretic background and the boson transformation formalism, we do not describe this approach here. It is best described in the review article of this theory by Wadati (1979). (See also: Umezawa et al 1982.) We mention also that Holz $(1979,1980)$ has given a microscopic Hamiltonian theory of dislocations in crystals.

\section{Conclusion}

The present review was intended to bring out the fact that there is as yet no single unified theory of all defects in crystals. The merits and drawbacks of the various theories available have been pointed out. To the reviewer the following areas seem to need urgent attention. The idea of Kröner (1970) that the loss of information in the coarse-graining process needed to define the dislocation-density tensor can be remedied with the help of higher-order correlation functions, needs to be pursued further. The alternate idea of Kröner and Teodosiu (1974) that it must be possible to describe the deformation behaviour of materials in terms of the fluctuations of the stress and strain (produced by dislocations) clearly suggests a stochastic approach to plastic flow. The difficulty here is the fact that even the deterministic equations needed to completely describe plastic flow are yet to be developed. In fact this was the spirit of the work of Kröner and Teodosiu (1974). Perhaps some extremely simple situations (like steady state creep) must be modelled first in terms of a stochastic description, and the relevant correlation tensors explicitly computed. Both Kosevich (1979) and Kröner and Teodosiu (1974) have proposed hydrodynamic equations for matter undergoing deformation. One testing ground for such hydrodynamic equations could be the workhardening behaviour of materials. Cottrell (1953) has given a beautiful analogy between stage I of work-hardening and the laminar flow of a fluid, and between stage II and turbulent flow. However, one does not yet know what the 'Reynold number' is which would characterize the transition to 'turbulence' in the case of plastic flow. In the spirit of this analogy, it may be possible to introduce different types of dislocation density tensors to describe the transition from stage I to stage II of the work-hardening curve. It seems that much work remains to be done in developing a hydrodynamic theory of a continuous medium containing dislocations.

\section{References}

Amari 1962 in RAAG memoirs of the unifying study of the basic probiems in engineering and physical sciences by means of geometry (ed) K Kondo (Tokyo: Gakujutsu Bunken Fukyu-kai), Vol 3, p 193 
Bilby B A 1960 in Progress in solid mechanics (eds) I N Sneddon and R Hill (Amsterdam: North-Holland), Vol 1, p 329

Bilby B A. Bullough R and Smith E 1955 Proc. R. Soc. (London) A231 263

Burgers J M 1939 Proc. Acad. Sci. Amsterdam 42378

Cartan E 1928 Lecons sur la Géométrie des espaces de Riemann (Paris: Gauthier-Villars)

Cottrell A H 1953 Dislocations and plastic flow in crystals (Oxford: Clarendon Press)

de Wit R 1960 in Solid state physics (eds) F Seitz and D Turnbull (New York: Academic) Vol 10, p 249

Dzyaloshinskii I and Volovik G 1978 J. Phys. 39693

Edelen D G B 1980 Int. J. Eng. Sci. 181095

Eringen A C 1972 lnt. J. Eng. Sci. 10425

Eringen A C 1973 Lett. Appl. Eng. Sci. 111

Eringen A C 1976 Continuum Physics, Vol IV, (New York: Academic)

Eringen A C 1977a J. Phys. D10 671

Eringen A C 1977b Int. J. Eng. Sci. 15177

Eringen A C and Balta F 1979 Crysial Lattice Defects 873

Eringen A C and Edelen D G 1972 Int. J. Eng. Sci. 10233

Eringen A C. Speziale C G and Kim B S 1977 J. Mech. Phys. Solids 25339

Eshelby J D 1956 in Solid state physics (eds) F Seitz and D Turnbull (New York: Academic) Vol 3, p. 79

Eshelby J D 1957 Proc. R. Soc. (London) A241 376

Eshelby J D 1961 in Progress in solid mechanics (eds) I N Sneddon and R Hill (Amsterdam: North-Holland) Vol 2, p 89

Evans J H, Bullough R and Stoneham A M 1972 in Radiation induced voids in metals (eds) J W Corbett and L C Ianiello USAEC-CONF-71061

Flinn P A and Maraduddin A A 1962 Ann. Phys. 1881

Frank F C 1951 Philos. Mag. 42809

Gairola B K D 1979 in Dislocations in solids (ed) F R N Nabarro (Amsterdam: North-Holland) Vol 1, p 223

Heald P T and Speight M V 1974 Philos. Mag. 291075

Holz A 1979 Phys. Ret. A20 2521

Holz A 1980 Phys. Rev. B22 3678

Julia B and Toulouse G 1979 J. Phys. 40 L-395

Kadić A and Edelen D G B 1982 Int. J. Eng. Sci. 20 49, 433, 747, (see also Kadić A and Edelen D G B 1983 A gauge theory of dislocations and disclinations (Berlin: Springer-Verlag))

Kondo K 1952 On the geometrical and physical foundations of theory of yielding in Proc. 2nd Japan Nat. Conyr. Applied Mechanics, Tokyo

Kondo K 1955 Non-Riemannian geometry of imperfect crystals from a macroscopic viewpoint, in $R A G G$ memoirs of the unified study of basic problems in engineering and physical sciences by means of geometry (ed) K Kondo (Tokyo: Gakujutsu Bunken Fukyu-kai)

Kosevich A M 1979 in Dislocations in solids (ed) F R N Nabarro (Amsterdam: North-Holland) Vol 1, p 33 Kovács I 1978 Physica B94 177

Kovács I and Vörös G 1979 Physica B96 111

Kröner E 1953 Z. Phys. 136402

Kröner E 1964 Phys. Kondens. Materie 2262

Kröner E 1970 in Inelastic behatiour of solids (eds) M F Kanninen, W F Adler, A R Rosenfeld and R I Jaffee (New York: McGraw-Hill) p 135

Kröner E 1981 in Les Houches Session XXXV, 1980- Physics of defects (eds) R Balian et al (Amsterdam: North-Holland)

Kröner and Teodosiu C 1974 in Problems of plasticity, Int. Symp. on Foundations of Plasticity, Warsaw 1972, (ed) A Sawczuk (Leyden: Noordhoff Intl Fublishing) p 45

Kroupa F 1962 Czech. J. Phy's. B12 191

Leibfried G and Breur N 1978 Point defects in metals (Berlin: Springer-Verlag) Vol I

Malen K and Bullough R 1971 in BNES European Conf, Reading, England, March 1971

Marcinkowski M J 1979 Unified theory of the mechanical behaviour of matter (New York: Wiley)

Mura T 1968 Adv. Mater. Res. 31

Nabarro F R N 1967 Theory of crystal dislocations (Oxford: Clarendon)

Noll W 1967 Arch. Rational Mech. Anal. 271

Nye J F 1953 Acta Metall. 1153

Peach M and Koehler J S 1950 Phys. Rev. 80436 
Schouten J A 1954 Ricci-Calculus (Berlin: Springer-Verlag)

Seeger A 1961 Phys. Status Solidi 1669

Siems R 1968 Phys. Status Solidi 30645

Steeds J W and Willis J R 1979 in Dislocations in solids (ed) F R N Nabarro (Amsterdam: North-Holland) Vol 1 p 33

Teodosiu C 1982 Elastic models of crystal defects (Berlin: Springer-Verlag)

Umezawa H Matsumoto H and Tachiki M 1982 Thermo field dynamics and condensed systems (Amsterdam: North-Holland)

Wadati M 1979 Phys. Rep. $\mathbf{5 0} 87$

Wadhawan V K 1982 Phase Transitions 33

Wang C C 1967 Arch. Rational Mech. Anal. 2733

Zorawski M 1967 Théorie mathématique des dislocations (Paris: Dunod)

\section{Discussion}

T V Ramakrishnan: What is the continuum local theory value for the self-energy of a point defect?

D Sahoo: For a mathematical point defect, it is infinity, but for a rigid 'point' defect occupying a volume $\Delta V$, it is $\frac{2}{3} \mu \Delta V$, where $\mu$ is the shear modulus of the medium.

N Kumar: What is $\boldsymbol{P}$ in the nonlocal theory? Can you go to the local limit through some approximation?

Sahoo: $\boldsymbol{P}$ is the elastic dipole tensor, as in the local theory. It is the interaction between a dipole and a strain field which is nonlocal. Yes, one can go to the local limit by the replacement $\alpha(R) \rightarrow \delta(R)$.

H R Krishna Murthy: Is the nonlocal theory supposed to simulate the lattice?

Sahoo: Yes, it is intermediate between the local continuum theory and the discrete atomistic theory. The lattice appears through the parameter $a$ occurring in the kernel $\alpha(R)$. This $a$ is essentially the lattice constant.

Ramakrishnan: In that case, where is the dispersion?

Sahoo: Eringen $(1972,1973)$ has explicitly calculated the dispersion for a onedimensional lattice. He has also calculated the dispersion curve for Rayleigh surface waves.

A S Parasnis: What exactly is it in the nonlocal theory that removes the infinities in the stress and strain at the core of a (screw) dislocation-a divergence which seems to be almost a necessity in a continuum theory?

Sahoo: It is essentially the replacement of all the $\delta$ functions by the finite kernel $\alpha(R)$. Being a smooth function like a Gaussian, this removes the infinities in the calculation of the integrals involved.

Parasnis: I have two comments: (i) I wonder if mere analogies, such as are implied in the attempt to have a 'unified' theory of defects, are likely to give physically significant theories and results. For example, two decades ago some mathematically-minded people drew an analogy between the closure failure $a$ la the Burgers/Frank circuit and Ampere's law and built up a fair edifice. But this could not explain the simple fact that like-currents attract whereas like-dislocations repel. What is needed is a physical theory 
going beyond such analogies. (ii) The difference between a Burgers circuit and a Frank circuit is that since the latter closes in the dislocated crystal/medium, it refers to the Burgers vector in the undislocated reference crystal/medium. You called the circuit a 'Frank circuit' but opened it in the dislocated crystal!

G Srinivasan: I wonder whether in the multiple glide region, the analogy may be with a cross-linked polymer rather than with turbulence. The problem in the polymer case has been made tractable by de Gennes, Edwards and others. The analogy may be worth pursuing.

C K Majumdar: Dr Srinivasan is apparently referring to the calculation of the elastic property of rubber by Prof. S F Edwards. There is a linear part, a plateau and a nonlinear rising part--somewhat similar to the regions I and II you refer to. It is quite possible that this analogy is better than that with laminar and turbulent flow. Now, the rubber and the polymer problems are dominated by entropy. Entropy is always difficult to handle in a mechanistic or hydrodynamic model. This difficulty appears also in the treatment of shocks or grain boundaries discussed earlier. The entropy equation, if written down from the second law, is somewhat different in nature from the hydrodynamic equations or the electrodynamic or the elastic equations (compare for example, the entropy generation equation of Sommerfeld). Edward's calculation of rubber elasticity is statistical mechanical in character, because entropy is involved. (Hydrodynamic equations or electrodynamic equations in continuous media are also statistical in character, but entropy is not so easily expressed in mechanical termsentropy has an integral character in phase space and is not easily expressed in a differential equation.) If the entropy equation could be written down, or rather, if the entropy could be woven into the framework, the analogy could be exploited-the insight of de Gennes and Edwards in random network problems may perhaps be useful here. 